\title{
Comparison for the Approximate Solution of the Second-Order Fuzzy Nonlinear Differential Equation with Fuzzy Initial Conditions
}

\author{
Ali F Jameel ${ }^{1, *}$, Akram H. Shather ${ }^{2}$, N.R. Anakira ${ }^{3}$, A. K. Alomari ${ }^{4}$, Azizan Saaban ${ }^{1}$ \\ ${ }^{1}$ School of Quantitative Sciences, College of Art and Sciences, Universiti Utara Malaysia (UUM), Malaysia \\ ${ }^{2}$ Department of Computer Engineering Techniques, Al-Kitab University Altun Kupri, Iraq \\ ${ }^{3}$ Department of Mathematics, Faculty of Science and Technology, Irbid National University, Jordan \\ ${ }^{4}$ Department of Mathematics, Faculty of Science, Yarmouk University, Jordan
}

Received April 13, 2020 ; Revised June 19, 2020; Accepted July 10, 2020

(a): [1] Ali F Jameel, Akram H. Shather, N.R. Anakira, A. K. Alomari, Azizan Saaban, "Comparison for the Approximate Solution of the Second-Order Fuzzy Nonlinear Differential Equation with Fuzzy Initial Conditions," Mathematics and Statistics, Vol. 8, No. 5, pp. 527 - 534, 2020. DOI: 10.13189/ms.2020.080505.

(b): Ali F Jameel, Akram H. Shather, N.R. Anakira, A. K. Alomari, Azizan Saaban (2020). Comparison for the Approximate Solution of the Second-Order Fuzzy Nonlinear Differential Equation with Fuzzy Initial Conditions. Mathematics and Statistics, 8(5), 527 - 534. DOI: 10.13189/ms.2020.080505.

Copyright $\mathrm{C} 2020$ by authors, all rights reserved. Authors agree that this article remains permanently open access under the terms of the Creative Commons Attribution License 4.0 International License

\begin{abstract}
This research focuses on the approximate solutions of second-order fuzzy differential equations with fuzzy initial condition with two different methods depending on the properties of the fuzzy set theory. The methods in this research based on the Optimum homotopy asymptotic method (OHAM) and homotopy analysis method (HAM) are used implemented and analyzed to obtain the approximate solution of second-order nonlinear fuzzy differential equation. The concept of topology homotopy is used in both methods to produce a convergent series solution for the propped problem. Nevertheless, in contrast to other destructive approaches, these methods do not rely upon tiny or large parameters. This way we can easily monitor the convergence of approximation series. Furthermore, these techniques do not require any discretization and linearization relative with numerical methods and thus decrease calculations more that can solve high order problems without reducing it into a first-order system of equations. The obtained results of the proposed problem are presented, followed by a comparative study of the two implemented methods. The use of the methods investigated and the validity and applicability of the methods in the fuzzy domain are illustrated by a numerical example. Finally, the convergence and accuracy of the proposed methods of the provided example are presented through the error estimates between the exact solutions
\end{abstract}

displayed in the form of tables and figures.

Keywords Fuzzy Set Theory, Fuzzy Differential Equations, Nonlinear Fuzzy Initial Value Problem, Optimum Homotopy Asymptotic Method (OHAM), Homotopy Analysis Method (HAM)

\section{Introduction}

As a mathematical model, a large number of dynamic real-life problems can be formulated in mathematical equations. These models may take the form of ordinary or partial differential equations. Fuzzy differential equations are an important tool to model a dynamical system when information about its behavior is inadequate. Fuzzy differential equations with fuzzy initial conditions appear when the modeling of these problems was imperfect and its nature is under uncertainty that involves fuzzy parameters that cannot be detected through ordinary measurement [1]. Thus, fuzzy differential equations are suitable mathematical models, these kinds of measurements in which there exist uncertainties or vagueness. These models are raised in many real-life applications such us, population models [2], physics [3], medicine [4], etc. Some of these 
fuzzy differential equations have been solved numerically such as first-order linear fuzzy initial value problems $[5,6]$. Also, some approximate methods are involved with an approximate solution of various types and order of fuzzy differential equations by Nedal et al [7] and Jameel et al [8].

Both HAM and OHAM are classified as approximate methods that have been used to solve differential equations approximately in various applications [9-14] that have many advantages such as solving the difficult nonlinear. It often helps an engineer or scientist to better understand a physical problem and can help to improve future procedures and designs for solving problems, also solve high order FIVPs directly without reducing it into first-order system, and determine the accuracy of the approximate solution without needing the exact solution especially the nonlinear equations [14]. Approximate methods like HAM and OHAM provide a simple way to ensure the convergence of a series solution that comes from the great freedom to choose proper base function approximating a nonlinear problem [15]. In addition, these methods have the flexibility to give an approximate and exact solution to both linear and nonlinear problems without any need for discretization and linearization as numerical methods, hence is free rounding off errors and does not require computer memory or time [16]. The HAM can rate the convergence of the solution, by using the advantages of h-curves adjusts the accuracy of the solution by finding the best values of the convergence control parameter [10]. The OHAM has a built-in convergence criteria similar to HAM but with a greater degree of flexibility [16]. Some approximate methods were used to solve problems in the second-order and above involving fuzzy initial values [17].

Our main motivation is to present a complete fuzzy analysis of the problem of second-order fuzzy initials, followed by a fuzzy analysis of OHAM and HAM, to obtain an approximate solution to the proposed problem and to present a comparative study of these methods in detail. This is the first attempt to present a comparative and analytical study of the approximate solution in the nonlinear second-order fuzzy initial value problem using HAM and OHAM to the best of our knowledge.

This research outline is as follows: The second-order nonlinear fuzzy initial value problem of second necessary fuzzy analysis details is recalled. An analysis and description of HAM and OHAM general formulas are presented in separate sections. In the section 'Illustration and Discussion', a numerical example is involved and results have been compared and displayed by our proposed methods. Finally, there is a summary that contains the conclusions of this paper.

\section{Fuzzy Analysis of Second-Order Fuzzy Nonlinear Initial Value Problems}

By following the defuzzification by Ali et al [19] we consider second-order nonlinear fuzzy Initial value problem:

$$
\begin{gathered}
\tilde{y}^{\prime \prime}(t)=\tilde{a} \tilde{y}^{\prime}(t)+\tilde{b} \tilde{y}(t)+\tilde{c} N\left(\tilde{y}^{\prime}(t), \tilde{y}(t)\right)+\tilde{d} w(t),(1) \\
y\left(t_{0}\right)=\alpha, \tilde{y}^{\prime}\left(t_{0}\right)=\beta
\end{gathered}
$$

for $t \geq t_{0}$. According to [19] the defuzzifcation and the fuzzy analysis of Eq. (1) with the fuzzy level set $r \in[0,1]$ is as follows:

$\tilde{y}(t)$ : is a fuzzy function [19] of the crisp variable $x$ such that $[\tilde{y}(t)]_{r}=\left[y(t)^{(r)}, \bar{y}(t)^{(r)}\right]$,

$\tilde{y}^{\prime}(t)$ : is the first order fuzzy Hukuhara-derivative [20] such that $\left[\tilde{y}^{\prime}(t)\right]_{r}=\left[\underline{y}^{\prime}(t)^{(r)}, \bar{y}^{\prime}(t)^{(r)}\right]$,

$\tilde{y}^{\prime \prime}(t)$ : is the second order fuzzy Hukuhara-derivative [20] such that $\left[\tilde{y}^{\prime \prime}(t)\right]_{r}=\left[\underline{y}^{\prime \prime}(t)^{(r)}, \bar{y}^{\prime \prime}(t)^{(r)}\right]$,

$\widetilde{N}$ : is fuzzy nonlinear function of the crisp variable $x$, the fuzzy variable $\tilde{y}$ and the fuzzy derivative $\tilde{y}^{\prime}$ such that:

$$
\left[\widetilde{N}\left(\tilde{y}(t), \tilde{y}^{\prime}(x)\right)\right]_{r}=\left[\underline{N}\left(t, \underline{y}, \underline{y}^{\prime}\right)^{(r)}, \bar{N}\left(t, \bar{y}, \bar{y}^{\prime}\right)^{(r)}\right],
$$

where the nonhomogeneous term is $[\widetilde{w}(t)]_{r}=$ $\left[\underline{w}(t)^{(r)}, \bar{w}(t)^{(r)}\right]$. The fuzzy coefficients in Eqs. (1) are triangular fuzzy numbers [21] that can be identify as:

$$
[\tilde{a}]_{r}=[\underline{a}, \bar{a}]_{r}, \quad[\tilde{b}]_{r}=[\underline{b}, \bar{b}]_{r}, \quad[\tilde{c}]_{r}=[\underline{c}, \bar{c}]_{r} \text { and }
$$
$[\tilde{d}]_{r}=[\underline{d}, \bar{d}]_{r}$ for all the fuzzy level set $r \in[0,1]$.

$\tilde{y}_{0}$ : is the first fuzzy initial condition triangular numbers denoted by $\left[\tilde{y}\left(t_{0}\right)\right]_{r}=\left[\underline{y}\left(t_{0}\right)^{(r)}, \bar{y}\left(t_{0}\right)^{(r)}\right]$, such that $\left[\tilde{\alpha}_{0}\right]_{r}=\left[\underline{\alpha}_{0}, \bar{\alpha}_{0}\right]_{r}$, finally:

$\tilde{y}^{\prime}$ : is the second fuzzy initial condition triangular numbers denoted by $\left[\tilde{y}^{\prime}\left(t_{0}\right)\right]_{r}=\left[\underline{y}^{\prime}\left(t_{0}\right)^{(r)}, \bar{y}^{\prime}\left(t_{0}\right)^{(r)}\right]$, such that $\left[\tilde{\beta}_{0}\right]_{r}=\left[\underline{\beta}_{0}, \bar{\beta}_{0}\right]_{r}$. For simplest form of Eq. (1) let

$$
\tilde{a} \tilde{y}^{\prime}(t)+\tilde{b} \tilde{y}(t)+\tilde{c} N\left(\tilde{y}^{\prime}(t), \tilde{y}(t)\right)+\tilde{d} w(t)=\tilde{f}(t, \tilde{y}(t))
$$

where

$$
\left\{\begin{array}{l}
f(t, \tilde{y}(t))^{(r)}=L\left[\underline{y}, \bar{y}, \underline{y}^{\prime}, \bar{y}^{\prime}\right]_{r} \\
\bar{f}(t, \tilde{y}(t))^{(r)}=U\left[\underline{y}, \bar{y}, \underline{y}^{\prime}, \bar{y}^{\prime}\right]_{r}
\end{array}\right.
$$

then Eq. (1) become as follows

$$
\tilde{y}^{\prime \prime}(t)=\tilde{f}(t, \tilde{y}(t))
$$

By using the fuzzy extension fuzzy principle properties, we can define the following membership function $[22,23]$ : 


$$
\left\{\begin{array}{l}
f(t, \tilde{y}(t))^{(r)}=\min \{\tilde{f}(t, \tilde{\mu}(r)) \mid \tilde{\mu}(r) \in \tilde{y}(t)\} \\
\bar{f}(t, \tilde{y}(t))^{(r)}=\max \{\tilde{f}(t, \tilde{\mu}(r)) \mid \tilde{\mu}(r) \in \tilde{y}(t)\}
\end{array}\right.
$$

where

$$
\left\{\begin{array}{l}
\underline{f}(t, \tilde{y}(t))^{(r)}=L^{*}[t, \tilde{y}(t)]_{r}=L^{*}\left(t, \tilde{y}(t)^{(r)}\right) \\
\bar{f}(t, y(t))^{(r)}=U^{*}[t, \tilde{y}(t)]_{r}=U^{*}\left(t, \tilde{y}(t)^{(r)}\right)
\end{array}\right.
$$

From Eqs. (5) and (6), we rewrite Eq. (1) as follows

$$
\begin{gathered}
\left\{\begin{array}{c}
\underline{y}^{\prime \prime}(t)^{(r)}=L^{*}\left(t, \tilde{y}(t)^{(r)}\right) \\
y\left(t_{0}\right)^{(r)}=\underline{\alpha}_{0}, \underline{y}^{\prime}\left(t_{0}\right)^{(r)}=\underline{\beta}_{0}
\end{array}\right. \\
\left\{\begin{array}{c}
\bar{y}^{\prime \prime}(t)^{(r)}=U^{*}\left(t, \tilde{y}(t)^{(r)}\right) \\
\bar{y}\left(t_{0}\right)^{(r)}=\underline{\alpha}_{0}, \bar{y}^{\prime}\left(t_{0}\right)^{(r)}=\bar{\beta}_{0}
\end{array}\right.
\end{gathered}
$$

\section{Analysis of Fuzzy Homotopy Analysis Method}

In the study of this section, we give details and structure of HAM for the approximate solution of high order FIVPs. The HAM is applied to approximately solve high order linear and nonlinear FIVP. Toward this end, we consider Eq. (7-8) followed by the defuzzification of Eq. (1) In Section 2 such that for all $r \in[0,1]$ and according to the HAM we can construct the following correction functions as follows

$$
\left\{\begin{array}{l}
\underline{y}^{\prime \prime}(t ; r)=L^{*}\left(t, \tilde{y}(t)^{(r)}\right) \\
\bar{y}^{\prime \prime}(t ; r)=U^{*}\left(t, \tilde{y}(t)^{(r)}\right)
\end{array}\right.
$$

Also, we can write Eq. (10) as follows

$$
\begin{gathered}
\left\{\begin{array}{l}
L^{*}\left(t, \tilde{y}(t)^{(r)}\right)=0 \\
U^{*}\left(t, \tilde{y}(t)^{(r)}\right)=0
\end{array}\right. \\
\mathcal{F}([\widetilde{\varnothing}(t ; p)])=\min \left\{L^{*}[\underline{\emptyset}(t ; p)]_{r}, U^{*}[\bar{\varnothing}(t ; p)]_{r}: \mu \mid \mu \in\right. \\
\left.\left[L^{*}\left(t, \tilde{y}(t)^{(r)}\right)\right]\right\}, \\
\mathcal{G}([\widetilde{\varnothing}(t ; p)])=\max \left\{L^{*}[\underline{\varnothing}(t ; p)]_{r}, U^{*}[\bar{\varnothing}(t ; p)]_{r}: \mu \mid \mu \in\right. \\
\left.\left[U^{*}\left(t, \tilde{y}(t)^{(r)}\right)\right]\right\},
\end{gathered}
$$

where $\mu$ represents the membership function of Eq. (1). The zero-order deformation equation can be written as:

$$
\left\{\begin{array}{l}
(1-p) \underline{\mathcal{L}}_{2}\left[\underline{\emptyset}(t ; p)-\underline{y}_{0}(t ; r)\right]=p \hbar H(t) \mathcal{F}([\widetilde{\varnothing}(t ; p)]) \\
(1-p) \overline{\mathcal{L}}_{2}\left[\bar{\varnothing}(t ; p)-\bar{y}_{0}(t ; r)\right]=p \hbar H(t) \mathcal{G}([\widetilde{\varnothing}(t ; p)])
\end{array}\right.
$$

According to [9], $p \in[0,1]$ is an embedding parameter, and $\hbar$ is nonzero convergence- control parameter. The $H(t)$ is an auxiliary function while the operators $\underline{\mathcal{L}}_{2}=\frac{\partial^{2}[\underline{\emptyset}(t ; p)]_{r}}{\partial t^{2}}$ and $\overline{\mathcal{L}}_{2}=\frac{\partial^{2} \bar{\emptyset}[(t ; p)]_{r}}{\partial t^{2}}$ are auxiliary linear operators. We can define the initial approximation $\left[\tilde{y}_{0}(t)\right]_{r}=\left[\underline{y}_{0}(t ; r), \bar{y}_{0}(t ; r)\right]$, as follows:

$$
\left\{\begin{array}{l}
\underline{y}_{0}(t ; r)=\underline{C}_{1}(r)+\underline{C}_{2}(r) t \\
\bar{y}_{0}(t ; r)=\bar{C}_{1}(r)+\bar{C}_{2}(r) t
\end{array}\right.
$$

where for all $r \in[0,1], \tilde{C}_{1}(r) \operatorname{and} \tilde{C}_{2}(r)$ are the constants that can be determined easily from the initial conditions in Eq. (7-8). It can be concluded that when $p=0$ and $p=1$ we have

$$
\left\{\begin{array} { l } 
{ [ \emptyset ( t ; 0 ) ] _ { r } = \underline { y } _ { 0 } ( t ; r ) , } \\
{ [ \overline { \emptyset } ( t ; 0 ) ] _ { r } = \overline { y } _ { 0 } ( t ; r ) . }
\end{array} \quad \left\{\begin{array}{l}
{[\emptyset(t ; 1)]_{r}=\underline{y}(t ; r),} \\
{[\bar{\emptyset}(t ; 1)]_{r}=\bar{y}(t ; r) .}
\end{array}\right.\right.
$$

Hence as $p$ increases from 0 to 1 the solution $[\emptyset(t ; p)]_{r},[\bar{\varnothing}(t ; p)]_{r}$ varies from the initial guess $\underline{y}_{0}(t ; r)$ and $\bar{y}_{0}(t ; r)$ to the solution $\underline{y}(t ; r)$ and $\bar{y}(t ; r)$. By expanding $[\underline{\emptyset}(t ; p)]_{r}$ and $[\bar{\varnothing}(t ; p)]_{r}$ as a Taylor series with respect to $p$, we can obtain

$$
\left\{\begin{array}{l}
{[\underline{\emptyset}(t ; p)]_{r}=\underline{y}_{0}(t ; r)+\sum_{m=1}^{\infty} \underline{y}_{m}(t ; r) p^{m}} \\
{[\bar{\emptyset}(t ; p)]_{r}=\bar{y}_{0}(t ; r)+\sum_{m=1}^{\infty} \bar{y}_{m}(t ; r) p^{m}}
\end{array}\right.
$$

where

$$
\left\{\begin{array}{l}
\underline{y}_{m}(t ; r)=\left.\frac{1}{m !} \frac{\partial^{m}[\emptyset(t ; p)]_{r}}{\partial p^{m}}\right|_{p=0} \\
\bar{y}_{m}(t ; r)=\left.\frac{1}{m !} \frac{\left[\partial^{m} \bar{\emptyset}(t ; p)\right]_{r}}{\partial p^{m}}\right|_{p=0}
\end{array}\right.
$$

If auxiliary linear operator $\tilde{\mathcal{L}}_{2}$, the initial guesses $\underline{y}_{0}(t ; r)$ and $\bar{y}_{0}(t ; r)$, the convergence control parameter $h$, and the auxiliary function $H(t)$, are properly chosen then the series (17) converges to the exact solution at $p=1$ and we have:

$$
\left\{\begin{array}{l}
{[\underline{\emptyset}(t ; 1)]_{r}=\underline{y}_{0}(t ; r)+\sum_{m=1}^{\infty} \underline{y}_{m}(t ; r)} \\
{[\bar{\emptyset}(t ; 1)]_{r}=\bar{y}_{0}(t ; r)+\sum_{m=1}^{\infty} \bar{y}_{m}(t ; r)}
\end{array}\right.
$$

According to [19] we define the vectors

$$
\left\{\begin{array}{l}
\vec{y}_{i}(t ; r)=\left\{\underline{y}_{0}(t ; r), \underline{y}_{1}(t ; r), \ldots, \underline{y}_{m}(t ; r)\right\} \\
\overline{\vec{y}}_{i}(t ; r)=\left\{\bar{y}_{0}(t ; r), \bar{y}_{1}(t ; r), \ldots, \bar{y}_{m}(t ; r)\right\}
\end{array}\right.
$$

Also according to Section 2 differentiating (17) $m$ times with respect to the embedding parameter $p$ and then setting $p=0$ and dividing them by $m$ !, we have the $m^{\text {th }}$ order deformation equation

$\left\{\begin{array}{l}\underline{\mathcal{L}}_{2}\left[\underline{y}_{m}(t ; r)-\chi_{m} \underline{y}_{m-1}(t ; r)\right]=h \mathcal{R}_{m}\left(\overrightarrow{\vec{y}}_{m-1}(t ; r)\right) \\ \overline{\mathcal{L}}_{2}\left[\bar{y}_{m}(t ; r)-\chi_{m} \bar{y}_{m-1}(t ; r)\right]=h \mathcal{R}_{m}\left(\overline{\vec{y}}_{m-1}(t ; r)\right)\end{array}\right.$

where

$$
\left\{\begin{array}{l}
\mathcal{R}_{m}\left(\underline{\vec{y}}_{m-1}(t ; r)\right)=\left.\frac{1}{(m-1) !} \frac{\partial^{m-1} \mathcal{F}([\widetilde{\varnothing}(t ; p)])}{\partial p^{m-1}}\right|_{p=0} \\
\mathcal{R}_{m}\left(\overline{\vec{y}}_{m-1}(t ; r)\right)=\left.\frac{1}{(m-1) !} \frac{\partial^{m-1} \mathcal{G}([\widetilde{\varnothing}(t ; p)])}{\partial p^{m-1}}\right|_{p=0}
\end{array}\right.
$$

The solution of the $m^{t h}$ order deformation for $m \geq 1$ is:

$$
\left\{\begin{array}{l}
\underline{y}_{m}(t ; r)=\chi_{m} \underline{y}_{m-1}(t ; r)+\hbar \underline{\mathcal{L}}^{-1} \mathcal{R}_{m}\left(\vec{y}_{m-1}(t ; r)\right) \\
\bar{y}_{m}(t ; r)=\chi_{m} \bar{y}_{m-1}(t ; r)+\hbar \overline{\mathcal{L}}_{2}{ }^{-1} \mathcal{R}_{m}\left(\overline{\vec{y}}_{m-1}(t ; r)\right)
\end{array}\right.
$$




$$
\chi_{m}= \begin{cases}0, & m \leq 1 \\ 1, & m>0\end{cases}
$$

where ${\underline{\mathcal{L}_{2}}}^{-1}=\iint\left[\underline{]}_{r} d t d t \text { and } \overline{\mathcal{L}}_{2}^{-1}=\iint \overline{[}_{r}\right]_{r} d t d t$ are the inverse fuzzy integral operators, $\forall r \in[0,1]$. Note that we can apply the convergence analysis of HAM that discussed in [24].

\section{Analysis of Fuzzy Optimal Homotopy Asymptotic Method}

This section presents the analysis of OHPM to obtain an approximate solution of second order nonlinear FIVP. According to Section 3, we fuzzify OHAM and then defuzzify for the approximate solution of Eq. (1). Again, consider Eqs. (7-8) followed by the defuzzification of Eq. (1) in Section 2 such that for all $r \in[0,1]$ we rewrite Eqs. (8-9) in the following forms

$$
\begin{aligned}
\underline{\mathcal{L}}_{2}(\underline{y}(t ; r))-L^{*}\left(t, \tilde{y}(t)^{(r)}\right) & =0, t \in\left[t_{0}, T\right] \\
\mathcal{B}\left(\underline{y}(t ; r), \frac{\partial[\underline{y}] r}{\partial t}\right) & =0
\end{aligned}
$$

and for the upper bound we have

$$
\begin{gathered}
\overline{\mathcal{L}}_{2}(\bar{y}(t ; r))-U^{*}\left(t, \tilde{y}(t)^{(r)}\right)=0, t \in\left[t_{0}, T\right] \\
\mathcal{B}\left(\bar{y}(t ; r), \frac{\partial[\bar{y}] r}{\partial t}\right)=0
\end{gathered}
$$

According to OHAM [25.26], Esq. (23-26) can also be written as follows:

$$
\begin{gathered}
(1-p)\left[\underline{\mathcal{L}}_{2}\left([\underline{\emptyset}(t ; p)]_{r}\right)\right]=\frac{\mathcal{H}(p ; r)\left[\underline{\mathcal{L}}_{2}\left([\underline{\emptyset}(t ; p)]_{r}\right)-\right.}{\left.\mathcal{F}\left([\widetilde{\varnothing}(t ; p)]_{r}\right)\right]} \\
(1-p)\left[\overline{\mathcal{L}}_{2}\left([\bar{\varnothing}(t ; p)]_{r}\right)\right]=\overline{\mathcal{H}}(p ; r)\left[\overline{\mathcal{L}}_{2}\left([\bar{\varnothing}(t ; p)]_{r}\right)-\right. \\
\left.\mathcal{G}\left([\widetilde{\varnothing}(t ; p)]_{r}\right)\right] \\
\mathcal{B}\left([\widetilde{\varnothing}(t ; p)]_{r}, \frac{\partial[\widetilde{\phi}(t ; p)]_{r}}{\partial t}\right)=0
\end{gathered}
$$

where $\mathcal{F}\left([\widetilde{\varnothing}(t ; p)]_{r}\right)$ and $\mathcal{G}\left([\widetilde{\varnothing}(t ; p)]_{r}\right)$ are defined in Eq.(12-13), $p \in[0,1]$ is an embedding parameter, and $\widetilde{\mathcal{H}}(p ; r)$ is a nonzero starting fuzzy function, for $p \neq 0$, and $\widetilde{\mathcal{H}}(0 ; r)=0,[\widetilde{\varnothing}(t ; p)]_{r}$ is an unknown fuzzy function, respectively. Now, when $p=0$ and $p=1$, we yield:

$$
\begin{cases}{[\emptyset(t ; 0)]_{r}=\underline{y}_{0}(t ; r),} & {[\underline{\emptyset}(t ; 1)]_{r}=\underline{y}(t ; r)} \\ {[\bar{\varnothing}(t ; 0)]_{r}=\bar{y}_{0}(t ; r),} & {[\bar{\varnothing}(t ; 1)]_{r}=\bar{y}(t ; r)}\end{cases}
$$

Thus, as $p$ increases from 0 to 1 , the solution $[\widetilde{\varnothing}(t ; p)]_{r}$ varies from $\tilde{y}_{0}(t ; r)$ to solution of Eqs. (28-30) denoted by $\tilde{y}(t ; r)$, for $p=0$ we have:

$$
\tilde{\mathcal{L}}_{2}\left(\tilde{y}_{0}(t ; r)\right)=0, \mathcal{B}\left(\tilde{y}_{0}(t ; r), \frac{\partial\left[\tilde{y}_{0}\right] r}{\partial t}\right)=0
$$

The popover starting function $\widetilde{\mathcal{H}}(p ; r)$ for equation $(28-30)$ in the form [13]:

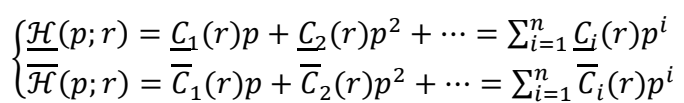

where $\tilde{C}_{1}(r), \tilde{C}_{2}(r), \ldots$ are the constants that become a function of $r$ to be found all $r \in[0,1]$. According to [24], expanding $\left[\widetilde{\varnothing}\left(t ; p, \check{C}_{i}(r)\right)\right]_{r}$ into Taylor's series about $p$, we obtain approximate solution series:

$$
\left[\widetilde{\varnothing}\left(t ; p, \check{C}_{i}(r)\right)\right]_{r}=\tilde{y}_{0}(t ; r)+\sum_{i=1}^{n}\left[\tilde{y}_{\mathrm{i}}\left(t, \check{C}_{i}(r)\right)\right]_{r} p^{i}
$$

Now, substitute Eq. (34) into Eqs. (28-29), and equating the coefficient of like powers of $p$ to obtain the following differential equations:

The zero ${ }^{\text {th }}$ order problem is given by:

$$
\begin{gathered}
\underline{\mathcal{L}}_{2}(\underline{y}(t ; r))-\underline{w}(t)^{(r)}=0, t \in\left[t_{0}, T\right] \\
\mathcal{B}\left(\underline{y}(t ; r), \frac{\partial[\underline{y}]_{r}}{\partial t}\right)=0
\end{gathered}
$$

and for the upper bound we have

$$
\begin{gathered}
\overline{\mathcal{L}}_{2}(\bar{y}(t ; r))-\bar{w}(t)^{(r)}=0, t \in\left[t_{0}, T\right] \\
\mathcal{B}\left(\bar{y}(t ; r), \frac{\partial[\bar{y}]_{r}}{\partial t}\right)=0
\end{gathered}
$$

First order Problem:

$$
\begin{aligned}
& \left\{\begin{array}{l}
\underline{\mathcal{L}}_{n}\left(\underline{y}_{1}(t ; r)\right)-\underline{w}(t ; r)=\underline{C}_{1}(r) \mathcal{F}_{0}\left(\tilde{y}_{0}(t ; r)\right) \\
\overline{\mathcal{L}}_{n}\left(\bar{y}_{1}(t ; r)\right)-\bar{w}(t ; r)=\bar{C}_{1}(r) \mathcal{G}_{0}\left(\tilde{y}_{0}(t ; r)\right)
\end{array}\right. \\
& \mathcal{B}\left(\tilde{y}_{1}(t ; r), \frac{\partial\left[\tilde{y}_{1}\right]_{r}}{\partial t}\right)=0
\end{aligned}
$$

Second order Problem

$$
\left\{\begin{array}{c}
\underline{\mathcal{L}}_{2}\left(\underline{y}_{2}(t ; r)\right)-\underline{\mathcal{L}}_{2}\left(\underline{y}_{1}(t ; r)\right)=\underline{C}_{2}(r) \mathcal{F}_{0}\left(\tilde{y}_{0}(t ; r)\right) \\
+\underline{C}_{1}(r)\left[\underline{\mathcal{L}}\left(\underline{y}_{n}\left(\underline{y}_{1}(t ; r)\right)+\mathcal{F}_{1}\left(\tilde{y}_{0}(t ; r), \tilde{y}_{1}(t ; r)\right)\right]\right. \\
\overline{\mathcal{L}}_{2}\left(\bar{y}_{2}(t ; r)\right)-\overline{\mathcal{L}}_{n}\left(\bar{y}_{1}(t ; r)\right)=\bar{C}_{2}(r) \mathcal{G}_{0}\left(\tilde{y}_{0}(t ; r)\right) \\
+\bar{C}_{1}(r)\left[\overline{\mathcal{L}}_{2}\left(\bar{y}_{1}(t ; r)\right)+\mathcal{G}_{1}\left(\tilde{y}_{0}(t ; r), \tilde{y}_{1}(t ; r)\right)\right] \\
\mathcal{B}\left(\tilde{y}_{2}(t ; r), \frac{\partial\left[\tilde{y}_{2}\right]_{r}}{\partial t}\right)=0
\end{array}\right.
$$

The general $n^{\text {th }}$ order formula with respect to $\tilde{y}_{n}(t ; r)$ is given by:

$$
\left\{\begin{array}{c}
\underline{\mathcal{L}}_{2}\left(\underline{y}_{n}(t ; r)\right)-\underline{\mathcal{L}}_{2}\left(\underline{y}_{n-1}(t ; r)\right)=\underline{C}_{n}(r) \mathcal{F}_{0}\left(\tilde{y}_{0}(t ; r)\right) \\
+\sum_{i=1}^{n-1} \underline{C}_{i}(r)\left[\underline{\mathcal{L}}_{2}\left(\underline{y}_{n-i}(t ; r)\right)+\mathcal{F}_{n-i}\left(\sum_{j=0}^{n-1} \underline{y}_{j}(t ; r)\right)\right] \\
\overline{\mathcal{L}}_{2}\left(\bar{y}_{n}(t ; r)\right)-\overline{\mathcal{L}}_{2}\left(\bar{y}_{n-1}(t ; r)\right)=\bar{C}_{n}(r) \mathcal{G}_{0}\left(\tilde{y}_{0}(t ; r)\right) \\
+\sum_{i=1}^{n-1} \bar{C}_{i}(r)\left[\overline{\mathcal{L}}_{2}\left(\bar{y}_{n-i}(t ; r)\right)+\mathcal{G}_{n-i}\left(\sum_{j=0}^{n-1} \bar{y}_{j}(t ; r)\right)\right]
\end{array}\right.
$$




$$
\mathcal{B}\left(\tilde{y}_{n}(t ; r), \frac{\partial\left[\tilde{y}_{n}\right]_{r}}{\partial t}\right)=0
$$

where $[\widetilde{w}(t)]_{r}$ is nonhomogeneous term of Eq.(1) which equal to zero if Eq.(1) is homogeneous iand $\mathcal{F}_{n-i}\left(\sum_{j=0}^{n-1} \tilde{y}_{j}(t ; r)\right)$ and $\mathcal{G}_{n-i}\left(\sum_{j=0}^{n-1} \tilde{y}_{j}(t ; r)\right)$ are the coefficient $p^{n}$ of in the expansion of $\mathcal{F}[\widetilde{\varnothing}(t ; p)]_{r}$ and $\mathcal{G}[\widetilde{\varnothing}(t ; p)]_{r}$ about the embedding parameter $p$

$$
\left\{\begin{array}{c}
\mathcal{F}\left(\left[\widetilde{\varnothing}\left(t ; p, \sum_{i=1}^{n} \bar{C}_{i}(r)\right)\right]_{r}\right)= \\
\mathcal{F}_{0}\left(\tilde{y}_{0}(t ; r)\right)+\sum_{n=1}^{\infty} F_{n}\left(\sum_{i=0}^{n}\left[\tilde{y}_{i}\right]_{r}\right) p^{n} \\
\mathcal{G}\left(\left[\widetilde{\varnothing}\left(t ; p, \sum_{i=1}^{n} \bar{C}_{i}(r)\right)\right]_{r}\right)= \\
\mathcal{G}_{0}\left(\tilde{y}_{0}(t ; r)\right)+\sum_{n=1}^{\infty} \mathcal{G}_{n}\left(\sum_{i=0}^{n}\left[\tilde{y}_{i}\right]_{r}\right) p^{n}
\end{array}\right.
$$

It has been observed that the convergence of the series (40) depends upon the auxiliary constants $\tilde{C}_{1}(r), \tilde{C}_{2}(r), \ldots$, , then at $p=1$, we obtain:

$$
\begin{gathered}
\tilde{y}_{*}\left(t, \sum_{i=1}^{n} \tilde{C}_{i}(r) ; r\right)=\tilde{y}_{0}(t ; r)+ \\
\left.+\sum_{i=1}^{n}\left[\tilde{y}_{i}\left(t, \sum_{i=1}^{n} \tilde{C}_{i}(r)\right)\right)\right]_{r}
\end{gathered}
$$

Substituting Eq. (41) into Eqs. (28-29) it results the following residual:

$$
\left\{\begin{array}{c}
\underline{\mathcal{R}}\left(t, \sum_{i=1}^{n} \underline{C}_{i}(r) ; r\right)=\underline{\mathcal{L}}_{2}\left(\underline{y}_{*}\left(t, \sum_{i=1}^{n} \underline{C}_{i}(r) ; r\right)\right)-\underline{w}(t ; r) \\
-\mathcal{F}\left(\tilde{y}_{*}\left(t, \sum_{i=1}^{n} \tilde{C}_{i}(r) ; r\right)\right) \\
\overline{\mathcal{R}}\left(t, \sum_{i=1}^{n} \bar{C}_{i}(r) ; r\right)=\overline{\mathcal{L}}_{2}\left(\bar{y}_{*}\left(t, \sum_{i=1}^{n} \bar{C}_{i}(r) ; r\right)\right)-\bar{w}(t ; r) \\
-\mathcal{G}\left(\tilde{y}_{*}\left(t, \sum_{i=1}^{n} \tilde{C}_{i}(r) ; r\right)\right)
\end{array}\right.
$$

Again as in previous sections if $\tilde{\mathcal{R}}=0$, then $\tilde{y}_{*}$ yields the exact solution, generally it doesn't happen, especially in nonlinear high order FIVPs problems. For the determinations auxiliary constants of $\tilde{C}_{i}(r), i=1,2 \ldots n$, we choose $t_{0}$ and $T$ regarding to Eq. (1) such that optimum values of $\widetilde{C}_{i}(r)$ for the convergent solution of the desired problem is obtained. To find the optimal values of $\tilde{C}_{i}(r)$ here, we apply the method of Least Squares as in [11] to obtain Eq. (42), where the residual $\tilde{\mathcal{R}}$ is given by

$$
\left\{\begin{array}{l}
{[\underline{\mathcal{R}}]_{r}=\underline{\mathcal{L}}_{2}\left(\left[{ }_{y_{*}}\right]_{r}\right)-\underline{w}(t ; r)-\mathcal{F}\left(\left[\tilde{y}_{*}\right]_{r}\right)} \\
{[\overline{\mathcal{R}}]_{r}=\overline{\mathcal{L}}_{2}\left(\left[\bar{y}_{*}\right]_{r}\right)-\bar{w}(t ; r)-\mathcal{G}\left(\left[\tilde{y}_{*}\right]_{r}\right)}
\end{array}\right.
$$

and

$$
\frac{\partial \tilde{S}}{\partial \tilde{C}_{1}(r)}=\frac{\partial \tilde{S}}{\partial \tilde{C}_{2}(r)}=\cdots \frac{\partial \tilde{S}}{\partial \tilde{C}_{n}(r)}=0
$$

\section{Illustration and Discussion}

Consider the second-order fuzzy nonlinear differential equation given by Jameel et al [27]:

$$
\begin{gathered}
y^{\prime \prime}(t)=-\left(y^{\prime}(t)\right)^{2}, \quad 0 \leq t \leq 0.1 \\
y(0)=(r, 2-r), y^{\prime}(0)=(1+r, 3-r), \forall r \in[0,1]
\end{gathered}
$$

It can be verified that by using the exact solution of Eq. (45) given in [27] such that

$$
\begin{gathered}
\underline{Y}(t ; r)=\ln \left[\left(e^{r}+e^{r} r\right) t+e^{r}\right], \bar{Y}(t ; r)=\ln \left[\left(3 e^{2-r}-\right.\right. \\
\left.\left.e^{2-r} r\right) t+e^{2-r}\right]
\end{gathered}
$$

\section{Fuzzy OHAM:}

Follow section 4 we have the followings:

Zero order problem:

$$
\tilde{y}_{0}{ }^{\prime \prime}(t ; r)=0
$$

$\tilde{y}_{0}(0 ; r)=[r, 2-r] \quad \tilde{y}_{0}{ }^{\prime}(0 ; r)=[(r+1),(3-r)]$,

First order problem:

$$
\begin{gathered}
\tilde{y}_{1}{ }^{\prime \prime}\left(t, \tilde{C}_{1}(r) ; r\right)=\left(1+\tilde{C}_{1}(r)\right) \tilde{y}_{0}{ }^{\prime \prime}(t ; r)+ \\
+\tilde{C}_{1}(r)\left(\tilde{y}_{0}{ }^{\prime}(t ; r)\right)^{2} \\
\tilde{y}_{1}(0 ; r)=0 \quad \tilde{y}_{1}{ }^{\prime}(0 ; r)=0
\end{gathered}
$$

Second order problem:

$$
\begin{gathered}
\tilde{y}_{2}{ }^{\prime \prime}\left(t, \tilde{C}_{1}(r), \tilde{C}_{2}(r) ; r\right)=\left(1+\tilde{C}_{1}(r)\right) \tilde{y}_{1}{ }^{\prime \prime}\left(t, \tilde{C}_{1}(r) ; r\right)+ \\
2 \tilde{C}_{1}(r) \widetilde{y}_{1}{ }^{\prime}\left(t, \tilde{C}_{1}(r) ; r\right) \tilde{y}_{0}{ }^{\prime}(t ; r)+\tilde{C}_{2}(r)\left(\tilde{y}_{0}{ }^{\prime \prime}(t ; r)+\right. \\
\left.\left(\tilde{y}_{0}{ }^{\prime}(t ; r)\right)^{2}\right) \\
\tilde{y}_{2}(0 ; r)=0 \quad \tilde{y}_{2}{ }^{\prime}(0 ; r)=0
\end{gathered}
$$

Third order problem:

$$
\begin{array}{r}
\tilde{y}_{3}{ }^{\prime \prime}\left(t, \tilde{C}_{1}(r), \tilde{C}_{2}(r), \tilde{C}_{3}(r) ; r\right)= \\
\left(1+\tilde{C}_{1}(r)\right) \tilde{y}_{2}{ }^{\prime \prime}\left(t, \tilde{C}_{1}(r), \tilde{C}_{2}(r) ; r\right)+ \\
\tilde{C}_{1}(r)\left[2 \widetilde{y}_{2}{ }^{\prime}\left(t, \tilde{C}_{1}(r), \tilde{C}_{2}(r) ; r\right) \tilde{y}_{0}(t ; r)+\left(\tilde{y}_{1}{ }^{\prime}\left(t, \tilde{C}_{1}(r) ; r\right)\right)^{2}\right]+ \\
\tilde{C}_{2}(r)\left(\tilde{y}_{1}{ }^{\prime \prime}\left(t, \tilde{C}_{1}(r) ; r\right)+\tilde{y}_{1}{ }^{\prime}\left(t, \tilde{C}_{1}(r) ; r\right) \widetilde{y}_{0}{ }^{\prime}(t ; r)\right)+ \\
\tilde{C}_{3}(r)\left(\tilde{y}_{0}{ }^{\prime \prime}(t ; r)+\left(\tilde{y}_{0}{ }^{\prime}(t ; r)\right)^{2}\right) \\
\tilde{y}_{3}(0 ; r)=0 \quad \tilde{y}_{3}{ }^{\prime}(0 ; r)=0
\end{array}
$$

Using Mathematica 11 DSolve Package to find the solutions for the lower and the upper bounds of Eqs. (46-49), we obtain

$$
\begin{array}{r}
\tilde{y}_{*}(t ; r)=\tilde{y}_{0}(t ; r)+\tilde{y}_{1}\left(t, \tilde{C}_{1}(r) ; r\right)+\tilde{y}_{2}\left(t, \tilde{C}_{1}(r), \tilde{C}_{2}(r) ; r\right)+ \\
\tilde{y}_{3}\left(t, \tilde{C}_{1}(r), \tilde{C}_{2}(r), \tilde{C}_{3}(r) ; r\right)
\end{array}
$$

According to Section 4 , the optimal values of $\tilde{C}_{1}(r)$, $\tilde{C}_{2}(r)$ and $\tilde{C}_{3}(r)$ can be determined from Eq. (44) as showing in the following tables below 
Table 1. Optimal values of $\underline{C_{1}}(r), \underline{C_{2}}(r)$, and $\underline{C_{3}}(r)$ given by 3 -terms of OHAM of Eq. (45).

\begin{tabular}{cccc}
\hline $\mathrm{r}$ & $\underline{C}_{1}(r)$ & $\underline{C}_{2}(r)$ & $\underline{C}_{3}(r)$ \\
\hline 0 & -0.9351596491831894 & -0.0006082988473094407 & -0.000006999389745013307 \\
\hline 0.2 & -0.9266645756783222 & -0.0007742120427816049 & -0.000010152801305786626 \\
\hline 0.4 & -0.9210977315678253 & -0.0008932128797667255 & -0.000012664712219154883 \\
\hline 0.6 & -0.9048425010620137 & -0.0012862461783431666 & -0.000022305602470522420 \\
\hline 0.8 & -0.8892228272285377 & -0.0017260290501527882 & -0.000035301876455202014 \\
\hline 1 & -0.8841507175930892 & -0.0018815459669624531 & -0.000040411173275566030 \\
\hline
\end{tabular}

Table 2. Optimal values of $\bar{C}_{1}(r), \bar{C}_{2}(r)$, and $\bar{C}_{3}(r)$ given by 3 -terms of OHAM of Eq. (45)

\begin{tabular}{cccc}
\hline $\mathrm{r}$ & $\bar{C}_{1}(r)$ & $\bar{C}_{2}(r)$ & $\bar{C}_{3}(r)$ \\
\hline 0 & -0.8390281381156103 & -0.0035238738570320164 & -0.00010888937024478792 \\
\hline 0.2 & -0.8458057432377328 & -0.0032486904248177180 & -0.00009567750340935401 \\
\hline 0.4 & -0.8503931605656544 & -0.0030679597757606305 & -0.00008736565633093382 \\
\hline 0.6 & -0.8644987095988346 & -0.0025409267849453480 & -0.00006483249923558979 \\
\hline 0.8 & -0.8791435465153755 & -0.0020410509805364498 & -0.00004591642229799645 \\
\hline 1 & -0.8841507175930892 & -0.0018815459669624531 & -0.00004041117327556603 \\
\hline
\end{tabular}

\section{Fuzzy HAM:}

According to Section 3 the starting function of Eq. (45) is taken as follows

$$
\left\{\begin{array}{l}
\underline{y}_{0}(t ; r)=r+(1+r) t \\
\bar{y}_{0}(t ; r)=(2-r)+(3-r) t
\end{array}\right.
$$

From to Section 3, we have:

$$
\begin{gathered}
\left\{\begin{array}{l}
\underline{y}_{m}(t ; r)=\chi_{m} \underline{y}_{m-1}(t ; r)+\hbar \underline{\mathcal{L}}_{2}^{-1} \mathcal{R}_{m}\left(\vec{y}_{m-1}(t ; r)\right) \\
\bar{y}_{m}(t ; r)=\chi_{m} \bar{y}_{m-1}(t ; r)+\hbar \overline{\mathcal{L}}_{2}{ }^{-1} \mathcal{R}_{m}\left(\overline{\vec{y}}_{m-1}(t ; r)\right)
\end{array}\right. \\
\underline{y}_{m}(0 ; r)=0, \bar{y}_{m}(0 ; r)=0, \underline{y}_{m}^{\prime}(0 ; r)=0, \bar{y}_{m}^{\prime}(0 ; r)=0
\end{gathered}
$$

where

$$
\left\{\begin{array}{c}
\mathcal{R}_{m}\left(\underline{\vec{y}}_{m-1}(t ; r)\right)= \\
\underline{y}_{m-1}^{\prime \prime}(t ; r)+\sum_{j=0}^{m-1} \underline{y}_{j}(t ; r) \underline{y}_{m-1-j}(t ; r) \\
\mathcal{R}_{m}\left(\overline{\vec{y}}_{m-1}(t ; r)\right)= \\
\bar{y}_{m-1}^{\prime \prime}(t ; r)+\sum_{j=0}^{m-1} \bar{y}_{j}^{\prime}(t ; r) \bar{y}_{m-1-j}{ }_{m-1}(t ; r)
\end{array}\right.
$$

From Eq. (45), we can obtain components of homotopy series solution for the lower and the upper bound of Eq. (45). It is to be noted that the series solution (24) depends upon the convergent control-parameter $h$. For a simple illustration as in [27], we plotted the $\hbar$-curve when $r=1$ for $\tilde{y}^{\prime}(0.01 ; h ; 1)$ to select the values of $h$ since that value of $r$ for the lower and upper solution of Eq. (45).

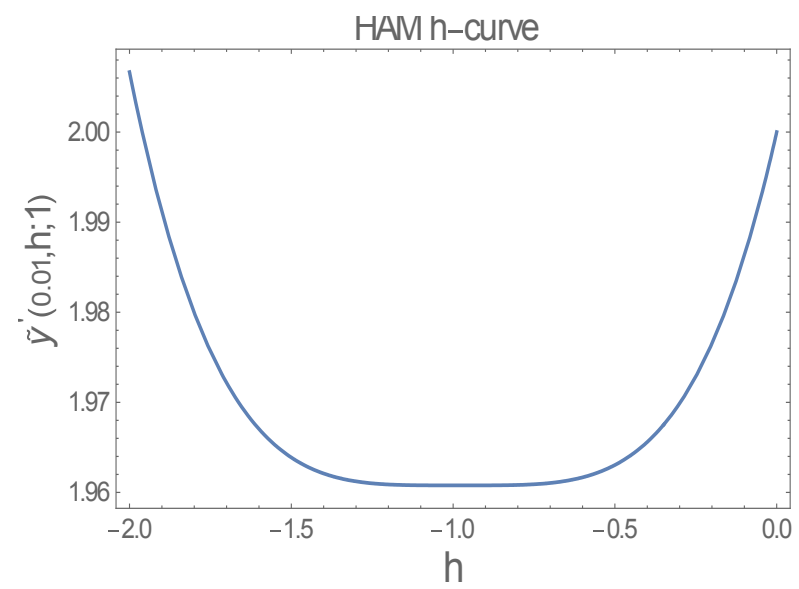

Figure 1. The h-curve of 4- terms Ham approximate solution of Eq. (45) at $r=1$.

According to [9] and Figure 1, the best convergent control-parameter $h=-0.9112$ is obtained to adjust the convergence region of the homotopy analysis solution for all $r \in[0,1]$.

Now we can tabulate the absolute errors $[E]_{r}$ and $[\bar{E}]_{r}$ of the approximate solutions $\underline{y}(0.1 ; r)$ and $\bar{y}(0.1 ; r)$ obtained by using 4-terms of HAM series solution for $h_{1}=-1$ and $h_{2}=-0.9112$ compared with 3-terms OHAM solution as follows: 


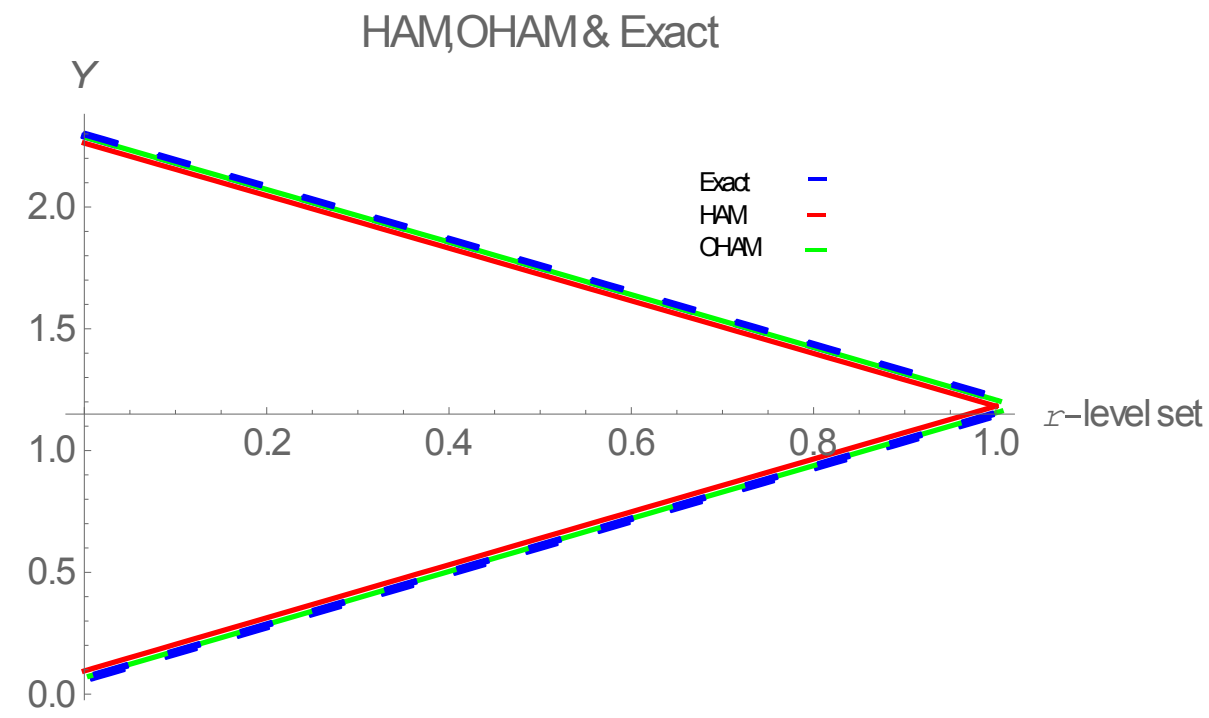

Figure 2. Compression of the approximate solution given by OHAM \& HAM with the exact solution of Eq. (45)]

Table 3. Comparison of the accuracy of the results solved by OHAM and HAM at $t=0.1$ for the lower solution of Eq. (45) for all $r \in[0,1]$

\begin{tabular}{cccc}
\hline $\mathrm{r}$ & {$[\underline{\mathrm{E}}]_{\mathrm{r}} h_{1} \mathrm{HAM}$} & {$[\underline{E}]_{r} h_{2} H A M$} & $\underline{E}(0.1 ; r) O H A M$ \\
\hline 0 & $1.53529 \times 10^{-7}$ & $3.98956 \times 10^{-8}$ & $1.59889 \times 10^{-10}$ \\
\hline 0.2 & $4.51332 \times 10^{-7}$ & $3.91122 \times 10^{-10}$ & $3.91122 \times 10^{-10}$ \\
\hline 0.4 & 0.0000011207 & $3.79328 \times 10^{-9}$ & $3.79328 \times 10^{-9}$ \\
\hline 0.6 & 0.0000024597 & $4.47279 \times 10^{-8}$ & $2.61947 \times 10^{-9}$ \\
\hline 0.8 & 0.0000049128 & $6.92457 \times 10^{-8}$ & $6.69669 \times 10^{-8}$ \\
\hline 1 & 0.00000910987 & $1.53606 \times 10^{-7}$ & $1.11097 \times 10^{-8}$ \\
\hline
\end{tabular}

Table 4. Comparison of the accuracy of the results solved by OHAM and HAM at $t=0.1$ for the upper solution of Eq. (45) for all $r \in[0,1]$

\begin{tabular}{cccc}
\hline $\mathrm{r}$ & {$[\bar{E}]_{r} h_{1} H A M$} & {$[\bar{E}]_{r} h_{2} H A M$} & $\bar{E}(0.1 ; r) O H A M$ \\
\hline 0 & 0.000096735 & 0.0000067076 & $1.26440 \times 10^{-7}$ \\
\hline 0.2 & 0.000064822 & 0.0000036619 & $9.18889 \times 10^{-8}$ \\
\hline 0.4 & 0.000042133 & 0.0000018656 & $7.34552 \times 10^{-8}$ \\
\hline 0.6 & 0.000026432 & $8.76047 \times 10^{-7}$ & $3.52946 \times 10^{-8}$ \\
\hline 0.8 & 0.000015907 & $3.76985 \times 10^{-7}$ & $1.51728 \times 10^{-8}$ \\
\hline 1 & 0.00000910987 & $1.53606 \times 10^{-7}$ & $1.11097 \times 10^{-8}$ \\
\hline
\end{tabular}

We can conclude from the above tables that the accuracy of the approximate solution solved by 3 -terms of OHAM is better than of 4-terms of HAM approximate solution at $\hbar=-0.9112$ and -1 for all $r \in[0,1]$ and $t \in$ $[0,0.01]$ of Eq. (45).

\section{Summary}

This study presented and applied two approximate analytical methods OHAM and HAM. These methods have been formulated under fuzzy set properties to obtain an approximate solution of second-order nonlinear FIVPs. OHAM and HAM show that the control and adjustment of the convergence of the series solution using the convergence-control parameters are achieved in a simple way. The comparison between OHAM and HAM shows that both methods are accurate and powerful in finding the solution of second-order nonlinear FIVP. Therefore, OHAM give a more accurate solution than HAM when $h=-1$ other values of $h$ for all fuzzy level sets with fewer terms. Moreover, HAM is faster than OHAM in terms of analysis and finding the approximate analytical solution of FIVPs because HAM needs less CPU time. One of the difficulties of OHAM in this study is that we need to determine the convergence control parameters for each $r$-level sets. The most disadvantage of OHAM is that this method includes many unknowns in some $r$-level set convergence-control parameters and this makes it time-consuming for calculating the approximate solution of FIVPs. Toward this end, the results obtained by OHAM and HAM are satisfied by the fuzzy number properties by taking either triangular fuzzy number shape.

\section{Acknowledgements}

This research was funded by a matching grant research collaboration project (S/O number 14130) between University Utara Malaysia, Sintok, Kedah, Malaysia, and Al-Kitab University, Kirkuk, Iraq.

\section{REFERENCES}

[1] Kaleva, O. (1987), Fuzzy differential equation, Fuzzy Sets Systems, 24, 301-317. 
[2] Smita, T., and Chakraverty, S. (2013), Numerical solution of fuzzy arbitrary order predator-prey equations, Applications and Applied Mathematics, 8(1), 647-673.

[3] El Naschie, M. S. (2005), From Experimental Quantum Optics to Quantum Gravity Via a Fuzzy Kahler Manifold, Chaos, Solitons \& Fractals, 25, 969-977.

[4] Abbod, M. F., Von Keyserlingk, D. G., and Mahfouf, M. (2001), Survey of utilization of fuzzy technology in medicine and healthcare, Fuzzy Sets Systems, 120, 331-3491.

[5] Allahviranloo, T., Ahmady, N., and Ahmady, E. (2007) Numerical solution of fuzzy differential equations by predictor-corrector method, 177(7) 1633-1647.

[6] Necdet. B., Asuman, A., and Sinan, Deniz. (2018) A New Approach to Fuzzy Differential Equations using Logarithmic Mean, Journal of Fuzzy Set Valued Analysis, 2018 (1), 10-21.

[7] Anakira, N. R., Shather, A. H., Jameel, A. F., Alomari, A. K., and Saaban, A. (2019) Direct solution of uncertain bratu initial value problem, International Journal of Electrical and Computer Engineering, 9(6), 221-240.

[8] Jameel, A. F., Anakira, N. R., Alomari, A. K., Mahameed, M. A., and Saaban, A. (2019), A New Approximate Solution of the Fuzzy Delay Differential Equations, International Journal Mathematical Modelling and Numerical Optimisation, 9(3), 221-240.

[9] Liao, S.-J. (1992) The Proposed Homotopy Analysis Techniques for The Solution of Nonlinear Problems, Ph.D. dissertation, Shanghai Jiao Tong University, Shanghai, China.

[10] Rashidi, M. M., Mohimanian pour, S. A., and Abbasbandy, S. (2011) 'Analytic approximate solutions for heat transfer of a micro polar fluid through a porous medium with radiation', Communications in Nonlinear Science and Numerical Simulation, 16, 1874-1889.

[11] Mabood, F., Khan, W.A., Ismail, A.I.M., 2013. Optimal homotopy asymptotic method for flow and heat transfer of a viscoelastic fluid in an axisymmetric channel with a porous wall. PLoS One, 8(12), e83581.

[12] Bogdan, M., Vasile,. M., (2018). Some exact solutions for MHD flow and heat transfer to modified second grade fluid with variable thermal conductivity in the presence of thermal radiation and heat generation/absorption. Computers \& Mathematics with Applications, 76(6), 1515-1524.

[13] M. Alipour, M. A. Vali, Appling Homotopy Analysis Method to Solve Optimal Control Problems Governed by Volterra Integral Equations, Journal of Computer Science \& Computational Mathematics, 5(3), 41-47, 2015.

[14] N. R. Anakira, A. F. Jameel, A. K. Alomari, A. Saaban, M. Almahameed I. Hashim, Approximate Solutions of Multi-Pantograph Type Delay Differential Equations Using
Multistage Optimal Homotopy Asymptotic Method, Journal of Mathematical and Fundamental Sciences, 50(3). 221-232, 2018.

[15] Liao, S.-J. (2004) 'On the homotopy analysis method for nonlinear problems', Applied Mathematics and Computation, 147(2), 499-513.

[16] Liang, S and Jeffrey, D. J. (2009) 'An efficient analytical approach for solving fourth order boundary value problems', Computer Physics Communications, 180, 2034-2040.

[17] Fazle, M., (2014). Comparison of optimal homotopy asymptotic method and homotopy perturbation method for non-linear equation. Journal of the Association of Arab Universities for Basic and Applied Sciences, 16(1), 21-26.

[18] Liao, S.-J. (1995) 'An approximate solution technique not depending on small parameters: a special example', International Journal of Non-Linear Mechanics, (3), 371380

[19] Ali, F. J., Azizan S., and Hamzeh, H. Z. (2018) Numerical solution of second-order fuzzy nonlinear two-point boundary value problems using combination of finite difference and Newton's methods', Neural Computing and Applications, 30(10), 3167-3175.

[20] Fard, O. S. (2009) 'An Iterative Scheme for the Solution of Generalized System of Linear Fuzzy Differential Equations', World Applied Sciences Journal, 7, 1597-11604.

[21] Guo, X., Shang, D and Lu, X. (2013) 'Fuzzy Approximate Solutions of Second-Order Fuzzy Linear Boundary Value Problems', Journal of Boundary Value Problems, 2013, pp. $1-17$.

[22] Dubois, D and Prade, H. (1982) 'Towards fuzzy differential calculus, Part 3: Differentiation', Fuzzy Sets and Systems, 8, 225-233.

[23] Zadeh, L. A. (2005) 'Toward A Generalized Theory of Uncertainty, Information Sciences', 172(2), 1-40.

[24] Ghoreishi, M., Ismail A., and Alomari, A. K. (2011) 'Application of the homotopy analysis method for solving a model for HIV infection of CD4+ T-cells', Mathematical and Computer modeling, 54, 3007-3015.

[25] Idrees, M, Islam, S, Tirmizi, S \& Haq, S. (2012), 'Application of the optimal homotopy asymptotic method for the solution of the Korteweg-de Vries equation', Mathematical and Computer Modelling, 55(3),1324-1333.

[26] Iqbal, S., Idrees, M., Siddiqui, A. M., and Ansari, A. R. (2010), 'Some solutions of the linear and nonlinear KleinGordon equations using the optimal homotopy asymptotic method', Applied Mathematics and Computation, 216(10), 2898-2909.

[27] Jameel, A.F., Ghoreishi, M. and Ismail, A. I. Md. (2014). Approximate Solution of High Order Fuzzy Initial Value Problems, journal of uncertain systems, 8(2),149-160. 